\title{
Battle of plates: a pilot study of an approach-avoidance training for overweight children and adolescents
}

\author{
Petra Warschburger ${ }^{1, *}$, Michaela Gmeiner ${ }^{1}$, Marisa Morawietz ${ }^{1}$ and Mike Rinck ${ }^{2}$ \\ 'Department of Psychology, University of Potsdam, Karl-Liebknechtstrasse 24-25, 14476 Potsdam, Germany: \\ ${ }^{2}$ Behavioural Science Institute, Radboud University of Nijmegen, Nijmegen, The Netherlands
}

Submitted 16 May 2017: Final revision received 8 August 2017: Accepted 17 August 2017: First published online 7 November 2017

\begin{abstract}
Objective: Approach-avoidance training (AAT) is a promising approach in obesity treatment. The present study examines whether an AAT is feasible and able to influence approach tendencies in children and adolescents, comparing implicit and explicit training approaches.

Design/Setting/Subjects: Fifty-nine overweight children and adolescents (aged 8-16 years; twenty-six boys) participated in an AAT for food cues, learning to reject snack items and approach vegetable items. Reaction times in the AAT and an implicit association test (IAT) were assessed pre- and post-intervention.

Results: A significant increase in the AAT compatibility scores with a large effect $\left(\eta^{2}=0 \cdot 18\right)$ was found. No differences between the implicit and explicit training approaches and no change in the IAT scores were observed.

Conclusions: Automatic tendencies in children can be trained, too. The implementation of AAT in the treatment of obesity might support the modification of an unhealthy nutrition behaviour pattern. Further data from randomized controlled clinical trials are needed.
\end{abstract}

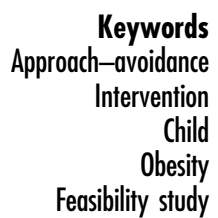

Obesity is a highly prevalent health problem. In Germany, $9 \cdot 6 \%$ of boys (11-17 years old) and $10.3 \%$ of girls are obese $^{(1)}$, with an incidence rate of $2.7 \%$ in school-aged children $^{(2)}$. There is substantial forward tracking of obesity into adulthood $^{(3)}$ and a positive relationship exists between childhood BMI and adult mortality ${ }^{(4)}$. The disease burden is tremendous and is expressed in manifold ways. Considerable evidence supports the association between childhood obesity and both medical (high blood pressure, diabetes type 2) and psychological problems like depression, eating disorders and low quality of life ${ }^{(5,6)}$. Additionally, childhood obesity is related to higher lifetime excess costs in adulthood ${ }^{(7)}$. Therefore, its successful and efficient treatment and its continuous improvement are essential topics.

Standard weight-loss treatments focus on modifying lifestyle factors like nutrition and physical activity. Besides initial positive effects on weight loss, their long-term success is limited ${ }^{(8)}$. Therefore, novel treatments are needed to increase their effectiveness, and focusing on the cognitive mechanisms underlying eating behaviour is one such compelling new approach. According to dual process models ${ }^{(9)}$, our behaviour is regulated via two processes or systems: the reflective and the impulsive system. Eating behaviours are largely considered to be automatic, involuntary behaviours triggered by internal (e.g. negative emotions) and external cues (e.g. availability of food). The strength of these 'obesogenic' eating patterns differs between normal- and overweight persons. For example, there is convincing evidence for enhanced reactivity to food stimuli in overweight compared with normal-weight individuals ${ }^{(10,11)}$. Thus, modifying these altered cognitive processes constitutes a promising approach in the design of novel intervention strategies because cognitive biases with respect to attention, associations and reactions could explain the gap between knowledge about healthy lifestyles and their implementation ${ }^{(12,13)}$.

One strategy in cognitive bias modification is the approach-avoidance training (AAT) ${ }^{(14)}$. AAT aims to modify automatic action tendencies by training participants to avoid tempting stimuli and approach alternative stimuli, typically by pushing and pulling a joystick. It has been shown that AAT successfully reduced relapse rates in alcohol-dependent patients ${ }^{(15,16)}$. In contrast, four recently published papers examining the effects of food-specific AAT in student samples yielded inconsistent results: three studies reported group differences with respect to approach-action tendencies after the training ${ }^{(17-19)}$, whereas one found no group differences ${ }^{(20)}$. Furthermore, while Schumacher et al. ${ }^{(19)}$ observed an effect on food 
consumption, Dickson et al. ${ }^{(17)}$ failed to find such an effect. Moreover, a recent uncontrolled study ${ }^{(21)}$ examined the effects of multisession AAT in a group of adults showing increased signs of eating pathology. A large significant reduction in approach-avoidance bias was reported and eating pathology scores dropped significantly between pre-treatment and the one-month follow-up. In conclusion, the training of approach-avoidance tendencies may be a promising method for bias modification $^{(22)}$, but previous results are less consistent compared with the alcohol domain ${ }^{(14)}$. This could be for different reasons: research in the eating domain did not focus on clinical samples, which show a stronger approach bias, but mainly on non-clinical samples (students within the normal weight range). In addition, compared with the eating domain more studies in the alcohol domain applied a multiple session design, which presumably resulted in a greater reduction of approach bias. Besides this, even though alcohol consumption and food intake share common features ${ }^{(23)}$, total abstinence of alcohol is the primary treatment goal. On the other hand, the establishment of healthy eating does not simply mean that snack foods should always be avoided. Moreover, there is no sharp distinction between 'healthy' and 'unhealthy' foods.

Besides approach-avoidance tendencies, automatic affective associations connected with a stimulus are a central component of the impulsive system ${ }^{(9)}$. A modification of automatic tendencies should therefore be accompanied by changes in affective associations. Since approach and association tendencies are both responses of the impulsive system $^{(9)}$, it is assumed that modifying approach tendencies will be accompanied by a change in the evaluation tendencies of the respective stimuli. Studies that revealed positive training effects for an AAT on alcohol approach bias reported such a generalization to implicit associations as measured with the implicit association test (IAT) ${ }^{(16,22)}$. In a similar manner, positive associations with unhealthy or high-energy foods were reported ${ }^{(20)}$. To the best of our knowledge, only one study has investigated the effects of a food-specific AAT on implicit food associations, finding no effect ${ }^{(18)}$.

To summarize, there is preliminary evidence for the efficacy of an AAT in modifying approach bias towards (unhealthy) foods. Overall, there appears to be a lack of studies in overweight samples, especially in children and adolescents. Previous studies were conducted with adult samples (mainly students), and it is not clear whether children and adolescents can be trained with the same paradigms. In addition, trainings targeting approachavoidance tendencies hardly ever employed explicit training instructions, which make the participants aware of the training goal. Instead, usually implicit training instructions, concealing the training goal, were used. There is some evidence that explicit and implicit training instructions yield similar treatment effects ${ }^{(16)}$. However, it is an open question whether this applies to children and adolescents as well. Furthermore, there are no data on how many sessions are needed to cause a training effect. Recent studies often used single-session trainings and focused predominantly on one food item (chocolate), whereas multisession trainings with a wide range of stimuli are considered to be a necessary component of successful trainings ${ }^{(24)}$. Prior to applying a randomized control group design, it is fundamental to test the feasibility of the training approach and the practicability of the implicit instructions. Therefore, the effects of an AAT were examined here in a group of overweight children and adolescents within a pilot study with a pre-post design. We expected that, at the end of the training, children and adolescents would show a training effect in their approach-avoidance tendencies. In addition, the potential generalization of the training to implicit associations with snacks was assessed. In an exploratory manner, we also looked to determine how many sessions were necessary to obtain a training effect. To evaluate the influence of the instruction mode, the children and adolescents were allocated to either an implicit or an explicit version of the AAT. In accordance with Wiers et al. ${ }^{(16)}$, we hypothesized that both training conditions would modify approach tendencies and implicit associations.

\section{Materials and methods}

\section{Participants}

Recruitment took place at two paediatric inpatient rehabilitation clinics. Comparable to other studies in the alcohol domain, the setting implemented the AAT in combination with treatment as usual: all patients participated in a lifestyle weight-loss intervention encompassing theoretical and practical lessons on physical activity and nutrition as well as a cognitive-behavioural group training. Children and adolescents aged between 8 and 16 years, overweight or obese (BMI > 90th percentile), not suffering from secondary obesity (obesity caused by a medical condition such as endocrine disorder or genetic syndrome) and not receiving physician-ordered pharmacological therapy for attentiondeficit hyperactivity disorder were eligible for participation. All participants received written, standardized information, participation was voluntary and they could withdraw approval at any time without disadvantages for their treatment. Written informed consent was obtained from the children and their parents. The study was approved by the local ethics committee.

\section{Sample characteristics}

Altogether, fifty-nine (thirty-three female) overweight or obese children and adolescents with a mean age of 13.23 (SD 1.93) years took part in the study. Before intervention, their mean BMI standard deviation score (BMI-SDS) reached 2.52 (SD 0.52), and $10 \cdot 2 \%$ were classified as 
overweight and $89.8 \%$ as obese. Based on the date of arrival, a cluster randomization was conducted: thirty participants were allocated to the explicit training condition, twenty-nine to the implicit training condition. There were no differences between the training groups in terms of gender $\left(\chi^{2}=1.36, P=0.24\right)$, age $(t(57)=0.37, P=0.72)$, BMI-SDS $(t(57)=1 \cdot 29, P=0 \cdot 20)$ or weight status $\left(\chi^{2}=0.82\right.$, $P=0.37)$.

\section{Procedure}

All participants were trained individually in six sessions over two weeks as a supplemental treatment offer by the clinics. The first four sessions were attended on a daily basis, followed by the remaining two sessions after a break of two or three days. Training sessions were implemented consistently, either after breakfast or after lunch. The training schedule was implemented for theoretical (distributed learning) and organizational reasons (no therapies on weekends). Every session contained the AAT, including training and test trials (approximately $15 \mathrm{~min}$ ). This training phase was framed by a short assessment of current hunger, appetite and mood, prior to and following the AAT. Additionally, each person completed the IAT in the first session (before the training) and the last session (after the training), these sessions lasting approximately 25-30 min.

\section{Measures}

Anthropometric data

The weight and height of each child were measured with a calibrated digital scale and a wall-mounted stadiometer. On that basis, the BMI-SDS was calculated ${ }^{(25)}$. The BMI-SDS indicates the difference of the individual's BMI compared with the average BMI of other children and adolescents of the same gender and age. According to national guidelines, overweight was defined as a BMI above the 90th percentile, obese as scores above the 97th percentile.

\section{Approach-avoidance training}

We decided to include vegetables instead of other sweet or savoury foods (such as fruits) to apply clearly distinct categories: fruits can also be considered as healthy in general, but consuming no more than two fruit portions daily is part of the education lessons for the participants of our study and we therefore did not want to confuse the children by training them to approach fruit snacks. Furthermore, vegetables in particular are even less frequently consumed than fruits, so there is a special need for promoting the consumption of vegetables ${ }^{(26)}$. For the AAT, we used twenty photographs of high-energy snacks (e.g. chocolate, muffins, popcorn, pretzel sticks, crackers) and twenty pictures of vegetables (e.g. tomatoes, cucumber, pepper, carrots, beetroot). We tested the familiarity and frequency of consumption, the categorization as 'healthy' or 'unhealthy', and the participants' willingness to taste that food item in a pilot study (conducted among 159 primary-school children). More than $98 \%$ of the food items were correctly categorized as 'healthy' or 'unhealthy'. Snack items were preferred and consumed slightly more often than vegetables. In the final picture set, only food items that were known and correctly named by at least $95 \%$ of the children were included. Food items were presented on either a circular or square plate. All items were matched according to their colour.

During the AAT, participants were instructed to either pull the pictures presented closer to them or push them away using the joystick. When the participants pulled the joystick towards them, the size of the picture on the screen increased, imitating an approach reaction. When the joystick was pushed away, the size decreased, imitating an avoidance reaction.

The AAT consisted of five phases: (i) a practice phase; (ii) a pre-assessment phase (sixty pictures of snacks, vegetables and empty plates); (iii) the actual training phase (120 pictures of snacks and vegetables); (iv) a postassessment phase (forty pictures of snacks and vegetables); and (v) the booster training phase (forty pictures of snacks and vegetables), which terminated the training session. Two different training conditions (implicit and explicit) were implemented. In both conditions, the participants were trained to pull the vegetables towards them and push the snack foods away, but the instructions differed. In the implicit condition, they were instructed to react to the shape of the plate (push square plates and pull circular plates). It was not mentioned during the training phases that the square plates were always filled with snacks and the circular ones with vegetables. In the explicit condition, children and adolescents were asked to react to the type of food (push snacks and pull vegetables). During the test blocks, all children and adolescents followed the implicit instructions. Participants' response latencies were recorded, and the lowest $1 \%$ and the highest $1 \%$ of the reaction times, as well as trials registering incorrect movements, were excluded ${ }^{(27)}$. Based on participants' mean response latencies, compatibility scores were calculated as the difference between the mean scores of 'approach-snack' and 'avoid-vegetable' stimulus-response combinations (pull snacks and push vegetables) minus 'avoid-snack' and 'approach-vegetable' ones (push snacks and pull vegetables) ${ }^{(20,22)}$. In addition, compatibility scores for snack stimuli and vegetable stimuli were calculated separately. For all scores, positive values indicate an effect corresponding to the intended training effect (more quickly pushing snacks and pulling vegetables).

\section{Implicit association test}

To measure implicit associations with snacks, participants completed a modified version of the single category picture IAT given by Karpinski and Steinman ${ }^{(28)}$ before and after the training. Stimuli consisted of seven pictures 
of high-energy snacks (drops, lollipops, gummy bears, pastries, ice cream, pretzels, nuts, crisps) ${ }^{(29)}$ and of seven positively (merry, great, fantastic, happiness, nice, peace, friend) and negatively (hate, disgusting, mean, pain, sad, misfortune, embarrassing) valenced words. To ensure that younger children with insufficient reading abilities could complete the test, verbal stimuli were provided via loudspeaker. The visual and auditory stimuli had to be sorted into one target category (snacks) and two attribute categories (good $v$. bad). We administered an IAT with four blocks: a practice block (twenty-four trials) followed by a congruent test block (seventy-two trials) and a practice block followed by an incongruent test block. In test blocks, the target category (snacks) was combined with either one of the attribute categories (good or bad) on one side (left or right) of the screen and the categorization responses were mapped to the same key. Based on the expected association, the block that required the same response for snacks and positive words was labelled the congruent block, while the block that required the same response for snacks and negative words was labelled the incongruent block. These blocks were presented in counterbalanced order.

To assess the strength of the implicit association, a difference score was calculated by subtracting the mean reaction time in the congruent block from the mean reaction time in the incongruent block. A positive score indicates a positive association with snacks and a negative score a negative association. The lowest $1 \%$ and the highest $1 \%$ of the reaction times and error trials were excluded $^{(28)}$.

\section{Mood, hunger and appetite}

To measure hunger ('How hungry are you right now?'), appetite ('How much do you feel like eating snacks/ vegetables right now?') and mood ('How do you feel right now?') as potential confounding factors, participants rated their current state on 5-point Likert scales ranging from 'not at all'/'very bad' (=1) to 'very much'/'very good' (=5) prior to and following the AAT in every single session.

\section{Acceptance}

To measure the acceptance of the AAT, participants rated their general liking of the training ('How much did you like the computer game?') after the last session on a 5 -point Likert scale ranging from 'not at all' $(=1)$ to 'very much' (=5).

\footnotetext{
Analyses

Our primary outcome measure was the change in action tendencies (measured with the AAT) over time. In the first step, we analysed data for potential confounding influences (age, gender, hunger, appetite, mood). Since no significant associations with our outcome measures were found, no covariates were included. To analyse the
}

general effectiveness of the training, we examined the AAT compatibility scores of the six sessions using a mixedfactor ANOVA with the between-subjects factor being training condition (implicit $v$. explicit instructions) and the within-subjects factor being time (with baseline and the six post-assessment points). In addition, we analysed the change across all sessions with a repeated-measure ANOVA including only the very first and the very last assessment. In addition to the overall compatibility scores, separate analyses were conducted for snacks and vegetables. As a measure of the magnitude of the treatment effects, the partial $\eta^{2}$ values are reported. According to Cohen ${ }^{(30)}$, partial $\eta^{2}$ values of about 0.01 can be classified as small, about 0.06 as medium, and values of 0.14 or greater as large effects. The course of the compatibility scores over the training sessions was analysed in an exploratory manner to determine the number of sessions necessary to create a training effect. In addition, each session was also analysed separately to test learning effects, comparing the compatibility scores at the beginning and at the end of a session. In general, conducting multiple tests yields the problem of cumulated $\alpha$ errors and usually requires an adjustment. According to Victor et $a l^{(31)}$, this is not recommended for exploratory procedures, therefore we decided not to adjust for multiple testing.

To evaluate changes in the IAT difference scores, another mixed-factor ANOVA with the between-subjects factor being training condition (implicit $v$. explicit instructions) and the within-subjects factor being time (before and after training) was performed. Acceptance of the AAT and its association with the training effect were analysed on a descriptive and correlational basis. All analyses were performed using the statistical software package IBM SPSS Statistics version 23. Significance was set at $P<0.05$ in all analyses.

\section{Results}

\section{Descriptive data}

Table 1 displays the performance data of the children and adolescents in the AAT and IAT at the beginning of the training. There were no significant differences between the training conditions.

\section{Approach-avoidance training}

Pre-post comparisons

The mixed-factor ANOVA (see Table 2 for details) including seven assessment points revealed a mediumsized, significant main effect of time on the compatibility scores. Neither the training group factor nor the interaction of time and training condition reached significance. Analysing the overall effect of the training including only the first and last assessment points revealed a large effect 
Table 1 Descriptive data at pre-test among fifty-nine overweight/obese children and adolescents aged 8-16 years (twenty-six boys), recruited in inpatient rehabilitation clinics in Germany (January-December 2014)

\begin{tabular}{|c|c|c|c|c|c|c|c|}
\hline & \multicolumn{6}{|c|}{ Training condition } & \multirow[b]{3}{*}{ Statistics } \\
\hline & \multicolumn{3}{|c|}{ Explicit ( $n$ 30) } & \multicolumn{3}{|c|}{ Implicit (n 29) } & \\
\hline & Mean & SD & Range & Mean & SD & Range & \\
\hline \multicolumn{8}{|c|}{ AAT compatibility score } \\
\hline Overall & $15 \cdot 9$ & $155 \cdot 2$ & $-350-483$ & 14.7 & $197 \cdot 7$ & $-303-495$ & $t(56)=0.02, P=0.981$ \\
\hline Snacks & -37 & $142 \cdot 1$ & $-560-184 \cdot 1$ & 1.0 & $125 \cdot 4$ & $-246 \cdot 8-220 \cdot 1$ & $t(56)=-1.08, P=0.286$ \\
\hline Vegetables & $-52 \cdot 8$ & 123.6 & $-223 \cdot 4-353.5$ & -3.7 & $147 \cdot 2$ & $-228 \cdot 6-315 \cdot 9$ & $t(57)=-1.39, P=0.17$ \\
\hline IAT difference score & -27 & $75 \cdot 2$ & $-200 \cdot 4-178 \cdot 2$ & $-25 \cdot 8$ & 99.1 & $-224 \cdot 2-156 \cdot 4$ & $t(56)=-0.05, P=0.958$ \\
\hline
\end{tabular}

AAT, approach-avoidance training; IAT, implicit association test.

Table 2 Pre-post comparisons of AAT compatibility scores by mixed-factor ANOVA among fifty-nine overweight/obese children and adolescents aged 8-16 years (twenty-six boys), recruited in inpatient rehabilitation clinics in Germany (January-December 2014)

\begin{tabular}{|c|c|c|c|c|c|c|}
\hline & \multicolumn{3}{|c|}{$\begin{array}{l}\text { Seven assessment } \\
\text { points }\end{array}$} & \multicolumn{3}{|c|}{$\begin{array}{l}\text { First and last assessment } \\
\text { points }\end{array}$} \\
\hline & $F$ & $\eta^{2}$ & $P$ & $F$ & $\eta^{2}$ & $P$ \\
\hline \multicolumn{7}{|c|}{ Compatibility scores for snacks and vegetables } \\
\hline Time & 6.5 & 0.11 & $<0.001$ & 12 & 0.18 & $<0.001$ \\
\hline Group & 0.33 & 0.01 & 0.5 & 0.8 & 0.01 & 0.375 \\
\hline Group & 0.9 & 0.02 & 3 & 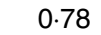 & 0.0 & 0.383 \\
\hline \multicolumn{7}{|c|}{ Compatibility scores for snacks } \\
\hline Time & 6.54 & 0.11 & $<0.001$ & $22 \cdot 6$ & 0.29 & $<0.001$ \\
\hline Group & 1.87 & 0.03 & & 0.75 & 0.01 & 0.39 \\
\hline Time $\times$ Group & 1.58 & 0.03 & 0.153 & $9 \cdot 14$ & 0.14 & $<0.001$ \\
\hline \multicolumn{7}{|c|}{ Compatibility scores for vegetables } \\
\hline Time & 4.44 & 0.08 & $<0.001$ & 0.39 & 0.08 & 0.537 \\
\hline Gro & 0.28 & 0.01 & & 0.2 & 0.00 & 0.66 \\
\hline Time $\times$ Group & $2 \cdot 12$ & 0.04 & 0.051 & $2 \cdot 32$ & 0.04 & $0 \cdot 133$ \\
\hline
\end{tabular}

AAT, approach-avoidance training.

of time (see Fig. 1), with no significant effects for either training condition or time $x$ training condition.

Separate analyses for the snack and vegetable compatibility scores confirmed the picture of the analyses reported above. With respect to the snack compatibility effect, we observed a significant main effect of time when including seven assessment points, as well as when including only the first and last assessments. The effect of the training condition was not significant in either one of the two analyses. A significant interaction effect was observed only when just two time points were included $v$. seven assessments. With respect to the vegetables compatibility effect, the main effect of time was significant when including all seven assessment points, but not with only two assessment points. There was neither a significant main effect of the training condition nor a significant time $\times$ training condition interaction in either analysis.

\section{Exploratory analyses of the training course}

On an exploratory level, we analysed the course of the overall compatibility scores over time through pairwise comparisons. Since we did not find a consistent influence of the training condition, this factor was no longer included in the analyses.

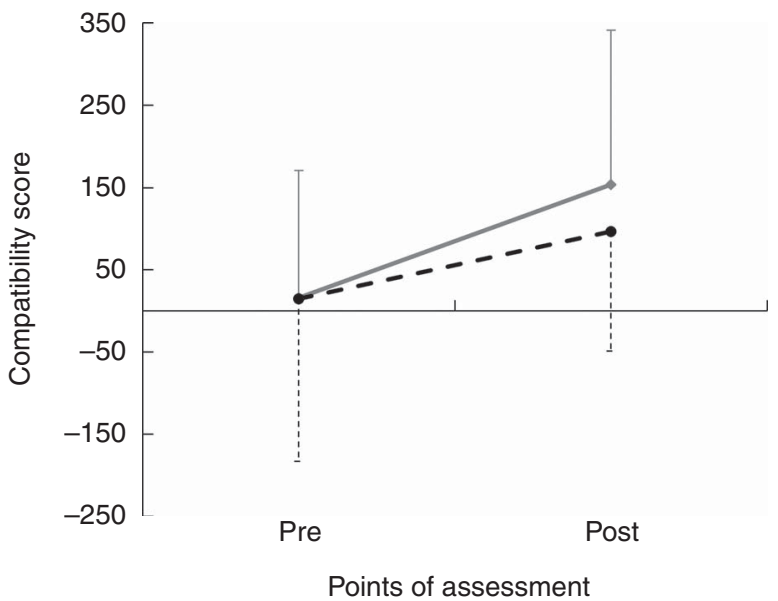

Fig. 1 Compatibility scores before (pre) and after (post) approach-avoidance training using implicit (-०-) and explicit $(\because)$ approaches among fifty-nine overweight/obese children and adolescents aged 8-16 years (twenty-six boys), recruited in inpatient rehabilitation clinics in Germany (January-December 2014). Values are means, with their standard deviations represented by vertical bars (for clarity, standard deviations are displayed in only one direction for each condition)

In a first step, we examined the difference between the pre-test score of the first session (initial baseline) and the post-training values of each session. At the end of each session, the compatibility score was significantly higher than at baseline (see Table 3).

In addition, the changes within each session were investigated. Significant increases in the compatibility scores were observed only for the first two sessions (see Table 3), with large effect sizes (session 1: $F(1,54)=$ 18.11, $P<0.001, \quad \eta^{2}=0.25$; session 2: $F(1, \quad 54)=9.98$, $\left.P=0 \cdot 003, \eta^{2}=0 \cdot 16\right)$.

\section{Implicit association test}

Contrary to our assumptions, our sample showed negative associations with snacks at pre-test (mean -26.4 (sD 87.5); $t(56)=-2 \cdot 28, P=0 \cdot 026)$. We observed neither a significant change over time $(F(1,54)=0 \cdot 14, P=0 \cdot 712)$, nor significant effects of the training condition $(F(1,54)=0 \cdot 08$, $P=0.773)$ or the interaction $(F(1,54)=0 \cdot 04, P=0 \cdot 837)$. Correlational analyses revealed a negative correlation 
Table 3 Mean compatibility scores over the AAT sessions and significance of changes among fifty-nine overweight/ obese children and adolescents aged 8-16 years (twenty-six boys), recruited in inpatient rehabilitation clinics in Germany (January-December 2014)

\begin{tabular}{|c|c|c|c|c|c|c|}
\hline \multirow[b]{3}{*}{ Session } & \multicolumn{4}{|c|}{ Mean compatibility scores } & \multicolumn{2}{|c|}{ Significance of changes } \\
\hline & \multicolumn{2}{|c|}{ Pre } & \multicolumn{2}{|c|}{ Post } & Difference within session* & \multirow{2}{*}{$\frac{\text { Difference from initial baseline } \dagger}{P}$} \\
\hline & Mean & SD & Mean & SD & $P$ & \\
\hline 1 & $7 \cdot 3$ & 174.5 & $171 \cdot 6$ & $206 \cdot 0$ & $<0.001$ & $<0.001$ \\
\hline 2 & 94.2 & 131.9 & $183 \cdot 2$ & 188.4 & 0.003 & $<0.001$ \\
\hline 3 & $143 \cdot 3$ & $190 \cdot 8$ & 177.6 & $179 \cdot 3$ & 0.258 & $<0.001$ \\
\hline 4 & 93.2 & 136.9 & $126 \cdot 9$ & 151.8 & 0.209 & $<0.001$ \\
\hline 5 & $86 \cdot 75$ & 162.5 & $132 \cdot 0$ & 205.4 & 0.184 & 0.002 \\
\hline 6 & $62 \cdot 8$ & $232 \cdot 2$ & $121 \cdot 3$ & $172 \cdot 1$ & 0.093 & 0.001 \\
\hline
\end{tabular}

AAT, approach-avoidance training.

${ }^{*}$ Change of scores within session from pre- to post-assessment.

†Change of baseline (pre-score of session 1) compared with the post-score of the particular session.

Table 4 Correlations of IAT difference scores with age and BMI-SDS among fifty-nine overweight/obese children and adolescents aged 8-16 years (twenty-six boys), recruited in inpatient rehabilitation clinics in Germany (January-December 2014)

\begin{tabular}{|c|c|c|c|c|c|}
\hline \multirow[b]{2}{*}{ Difference score } & & \multicolumn{2}{|c|}{ Age } & \multicolumn{2}{|c|}{ BMI-SDS } \\
\hline & & $r$ & $P$ & $r$ & $P$ \\
\hline IAT & $\begin{array}{l}\text { Pre } \\
\text { Post }\end{array}$ & $\begin{array}{l}0.03 \\
0.125\end{array}$ & $\begin{array}{l}0.85 \\
0.349\end{array}$ & $\begin{array}{r}0.20 \\
-0.02\end{array}$ & $\begin{array}{l}0.139 \\
0.861\end{array}$ \\
\hline
\end{tabular}

IAT, implicit association test; BMI-SDS, BMI standard deviation score.

between the post-training compatibility scores and the post-training IAT scores $(r=-0.29, P=0.03)$, indicating that more positive associations with snacks in the IAT went along with a stronger approach to snacks in the AAT. No significant correlations were found with age or with BMI-SDS (see Table 4).

\section{Additional analyses}

In addition, we observed no significant correlations of compatibility scores (pre, post, change) with age or BMI-SDS (see Table 5). The mean liking score of the training was 2.46 (SD 1.04), indicating an acceptance of the AAT in the middle range. There were no significant differences between boys and girls $(t(57)=-0.57, P=0.6)$, but between training conditions $(t(57)=2 \cdot 14, P=0 \cdot 037)$, with higher acceptance of the explicit condition (mean 2.73 (sD 0.98)) than of the implicit condition (mean 2.17 (SD 1.04)). We found no correlation of the acceptance scores with age $(r=-0 \cdot 2, P=0 \cdot 127)$. There was a significant correlation between the acceptance scores and the change in the compatibility scores, i.e. higher acceptance was associated with a more pronounced rise in the compatibility score (see Table 5).

\section{Discussion}

The primary aim of our study was to explore the trainability of action tendencies towards vegetables and snack
Table 5 Correlations of age, BMI-SDS and acceptance with AAT compatibility scores among fifty-nine overweight/obese children and adolescents aged 8-16 years (twenty-six boys), recruited in inpatient rehabilitation clinics in Germany (January-December 2014)

\begin{tabular}{|c|c|c|c|c|c|c|}
\hline & \multicolumn{2}{|c|}{$\begin{array}{l}\text { Pre-compatibility } \\
\text { score }\end{array}$} & \multicolumn{2}{|c|}{$\begin{array}{l}\text { Post- } \\
\text { compatibility } \\
\text { score }\end{array}$} & \multicolumn{2}{|c|}{$\begin{array}{l}\Delta \text { compatibility } \\
\text { scores }\end{array}$} \\
\hline & $r$ & $P$ & $r$ & $P$ & $r$ & $P$ \\
\hline Age & 0.07 & 0.593 & -0.21 & 0.116 & -0.20 & 0.135 \\
\hline BMI-SDS & -0.25 & 0.063 & 0.02 & 0.891 & 0.19 & 0.165 \\
\hline Acceptance & -0.20 & 0.131 & 0.23 & 0.085 & 0.30 & 0.021 \\
\hline
\end{tabular}

BMI-SDS, BMI standard deviation score; AAT, approach-avoidance training; $\Delta$ compatibility scores, post-compatibility score - pre-compatibility score.

foods by means of an AAT in overweight children and adolescents. Our second aim was to explore whether the changes in action tendencies were accompanied by changes in implicit associations. At an exploratory level, we addressed differences between implicit and explicit instructions and the number of sessions needed to elicit a significant training effect. To the best of our knowledge, the present study is the first to examine a food-specific AAT in a group of overweight children and adolescents.

As hypothesized, the children and adolescents successfully learned to alter their automatic tendencies. In addition, our results show that the AAT training was well accepted, with slightly higher acceptance scores for the explicit instruction. This might be due to the fact that this version offers a more varied game, since instruction formats have to change between training and testing phases. We did not find an influence of gender or age, suggesting that the training programme is applicable regardless of age or gender.

There is already evidence for a successful implementation of an AAT in this age group, focusing on anxiety in pre-adolescent children ${ }^{(32)}$ and adolescent smokers ${ }^{(33)}$. In line with these studies, our results underscore that automatic approach tendencies are trainable in children and adolescents, too. Up to now, current studies in the 
context of nutrition behaviour have solely focused on normal-weight adults, mainly students, and the pattern of results was inconsistent. In line with reports from four other studies $^{(17-19,21)}$, we found a significant change in automatic approach-avoidance tendencies, with effect sizes in the medium-to-large range, whereas Becker et $a{ }^{(20)}$ failed to find such a training effect in three experimental studies. Despite the fact that all of the studies mentioned above examined the effects of a food-specific AAT, they differ in many respects (such as training condition or stimulus material), aggravating the comparison. Due to the lack of a control group, one might overestimate the results; however, two other recent controlled studies ${ }^{(18,20)}$ reported similar results. One relevant factor explaining the controversial findings might be the stimulus materials or comparisons included. Our separate analyses for snacks and vegetables revealed significant changes in action tendencies for both stimuli, but less so for vegetables: effect sizes were lower than for snacks, and the analyses for vegetables were significant only when including all six post-intervention scores. This indicates that the training may be more effective for increasing avoidance of snacks, while effects on the approach of vegetable stimuli are less convincing. This is in line with studies showing trainability for the avoidance of unhealthy choices (mainly chocolate), but not for the approach of healthy choices $(15,16,20,22)$. Our results extend previous research with respect to the stimulus material, by applying a snack food $v$. vegetables paradigm. Regarding the trainability of approach reactions, only Maas et al. ${ }^{(18)}$ were able to observe such an effect (with e.g. a bell pepper, cucumber and apple as low-energy food stimuli and chocolate, French fries and hamburgers as high-energy food stimuli). In addition, moderating or mediating influences might play a role. Some authors ${ }^{(15)}$ argue that the stimulus materials must be salient in order to elicit training effects. This is in line with our data with respect to the acceptance of the presented stimulus materials. Vegetables are less well liked than snack foods and that could be an explanation for their lack of trainability. In general, it should be noted that our results apply to the comparison of snack foods and vegetables and are therefore not fully comparable with previous results. We did not observe significant training effects on implicit associations. The evidence on the effect to implicit associations is indeed quite inconsistent: positive associations were mainly reported in the context of alcohol drinking ${ }^{(16,22)}$, whereas for food-related stimuli, mainly non-significant effects were reported ${ }^{(18,20)}$. One possible explanation for this might be that the category of 'snacks' is fuzzier than the category of 'alcoholic beverages', the latter of which can be classified more easily.

Snack items are popular among children and adolescents and are consumed quite often ${ }^{(34)}$. Thus, one would expect that particularly overweight children and adolescents would exhibit a strong preference for snack items ${ }^{(35)}$. However, we observed unexpected negative associations with snacks at the beginning of the training. Analogously, some studies reported greater choices of healthy foods in youngsters ${ }^{(36)}$ and implicit negative associations with snacks in obese adults ${ }^{(37)}$. In accordance with Maas et $a l .{ }^{(18)}$, one might speculate that the clinical setting of our study goes along with a strong weight-loss control motivation of the obese children and adolescents, and this might serve as a buffer against the temptations of snack foods. From a statistical point of view, the already negative associations with snack foods might have prevented us from inducing even more pronounced negative associations. Future studies with larger sample sizes should analyse data on the basis of the initial implicit associations.

Despite the general lack of a generalization effect, we observed a negative correlation of the AAT compatibility score with the IAT score, indicating that the tendency to avoid snacks and to approach vegetables was associated with more pronounced negative associations with snacks. Because this relationship existed only after, but not before the training, an influence of the AAT may be present, which should be studied further in future studies.

In line with other studies ${ }^{(16)}$, our results do not indicate differences between the two instruction formats: implicit $v$. explicit. Only when we included just the pre- and posttraining data there was a significant time $\times$ instruction interaction for snacks. When examining the whole course of the training with seven assessments, the interaction effect was no longer significant. These preliminary data suggest that there may be no difference in treatment efficacy. Due to the fact that an implicit training version is more closely related to the basic idea of AAT, which is to modify automatic, implicit approach tendencies, it might be more adequate to apply the implicit training instruction. Regarding the number of sessions needed to elicit a reliable training effect, our data suggest that two sessions might be sufficient: after two, we observed a consolidation of the effect. This result contradicts that reported by Eberl et $a l .{ }^{(38)}$, who detected the strongest effects after six sessions. Therefore, the number of sessions necessary should be further explored in future studies. It must be considered that two sessions might be sufficient to show an effect of the training, but this is not automatically connected with a clinical effect (e.g. weight loss or changes in eating behaviour). Since we did not systematically vary the number of training sessions and only assessed the learning effect from session to session, it is premature to draw any conclusions with respect to the number of training sessions needed to elicit a 'clinically meaningful' training effect. Moreover, our results only pertain to a training effect in the AAT itself. Due to the exploratory nature of our multiple testing format, the risk of false positive conclusions can arise, and therefore our data should be interpreted with caution. Six sessions presenting the same content might become boring and decrease the 
recipients' interest over time. We cannot rule out the fact that a loss of interest in the training programme might influence the subsequent learning curve. In general, a gamification of the AAT for children is suggested ${ }^{(39)}$ to increase their motivation and acceptance. To ensure their motivation, continuous feedback on the points already earned combined with reinforcement strategies (e.g. smileys) might be helpful tools in this context. Taken together, our results provide preliminary evidence that food-related approach-avoidance tendencies can be successfully modified. Further research is needed to examine not only the feasibility of AAT but also its efficacy in clinical and non-clinical settings.

\section{Strengths and limitations}

On the one hand, several limitations of the current study should be pointed out. First, we did not include a placebo training control group. This means that we cannot conclude definitively that our observed changes over time are caused by our training programme. Second, our pilot study contained only pre- and post-assessments. We have no further information about the stability of the observed changes later on. Third, our sample is quite small and allocation to the two instruction modes was based on the participants' arrival in the clinic. In addition, we have no data on whether the training was associated with a change in the nutritional behaviour of the children and adolescents, or on their explicit liking of the food items. Since we compared snack foods with vegetables, we cannot draw any conclusion about the effects of a snack $v$. fruit comparison, for example. Future research on that topic is warranted.

On the other hand, the strengths of our study should be mentioned, too. First, we conducted a multisession training programme, recently recommended ${ }^{(38)}$ as a necessary ingredient of a successful training. Second, we considered different instruction conditions, and we included a variety of snack and vegetable items, increasing the external validity of our approach. Third, we included children and adolescents who were already overweight from two clinical settings, increasing the generalizability of our results to the relevant clinical target group of interest. Fourth, anthropometric data were objectively assessed, avoiding the problem of invalid self-report data in this sensitive field ${ }^{(40)}$. Finally, by including multiple outcome measures, we can add to the current knowledge on the generalizability of training effects.

\section{Conclusion}

In summary, our results showed that AAT is applicable in overweight children and adolescents. Not only is it accepted by the participants, but it is also associated with the expected positive effects. We did not find notable differences between an implicit and an explicit training condition, suggesting that even children can profit from the implicit version of the AAT.
Further studies including longer follow-up assessments and control groups are needed.

\section{Acknowledgements}

Acknowledgements: Special thanks are extended to the participating rehabilitation clinics, Spessartklinik Bad Orb and AHG Klinik Beelitz-Heilstätten, and the children and adolescents who volunteered for this trial. Thanks also to K. Lieck for programming the computer tasks. Financial support: This work was supported by the German statutory pension insurance scheme under Grant 8011-106-31/31.113. The funding organization played no role in designing and conducting the study, the data collection, its analysis and interpretation. Conflict of interest: None. Authorship: P.W. designed the study; M.R. developed the AAT and supported the data analysis; M.G. and P.W. were responsible for the statistical analysis; M.M. conducted the trainings. All authors took an active part in writing the paper. Ethics of buman subject participation: This study was conducted according to the guidelines laid down in the Declaration of Helsinki and all procedures involving human subjects/patients were approved by the Ethics Committee of the University of Potsdam, Germany. Written informed consent was obtained from all patients and their parents.

\section{References}

1. Brettschneider A-K, Schaffrath Rosario A, Kuhnert R et al. (2015) Updated prevalence rates of overweight and obesity in 11- to 17-year-old adolescents in Germany. Results from the telephone-based KiGGS Wave 1 after correction for bias in self-reports. BMC Public Health 15, 1101.

2. von Kries R, Beyerlein A, Müller MJ et al. (2012) Different age-specific incidence and remission rates in pre-school and primary school suggest need for targeted obesity prevention in childhood. Int J Obes (Lond) 36, 505-510.

3. Llewellyn A, Simmonds M, Owen CG et al. (2016) Childhood obesity as a predictor of morbidity in adulthood: a systematic review and meta-analysis. Obes Rev 17, 56-67.

4. Park MH, Falconer C, Viner RM et al. (2012) The impact of childhood obesity on morbidity and mortality in adulthood: a systematic review. Obes Rev 13, 985-1000.

5. Finkelstein EA, Graham WCK \& Malhotra R (2014) Lifetime direct medical costs of childhood obesity. Pediatrics $\mathbf{1 3 3}$, 854-862.

6. Sanders RH, Han A, Baker JS et al. (2015) Childhood obesity and its physical and psychological co-morbidities: a systematic review of Australian children and adolescents. Eur J Pediatr 174, 715-746.

7. Sonntag D, Ali S, Lehnert T et al. (2015) Estimating the lifetime cost of childhood obesity in Germany: results of a Markov model. Pediatr Obes 10, 416-422.

8. Altman M \& Wilfley DE (2014) Evidence update on the treatment of overweight and obesity in children and adolescents. J Clin Child Adolesc Psychol 44, 521-537.

9. Strack F \& Deutsch R (2004) Reflective and impulsive determinants of social behavior. Pers Soc Psychol Rev 8, 220-247.

10. Hendrikse JJ, Cachia RL, Kothe EJ et al. (2015) Attentional biases for food cues in overweight and individuals with obesity: a systematic review of the literature. Obes Rev 16, 424-432. 
11. Werthmann J, Jansen A \& Roefs A (2015) Worry or craving? A selective review of evidence for food-related attention biases in obese individuals, eating-disorder patients, restrained eaters and healthy samples. Proc Nutr Soc 74, 99-114.

12. Jansen A, Houben K \& Roefs A (2015) A cognitive profile of obesity and its translation into new interventions. Front Psychol 6, 1807.

13. Turton R, Bruidegom K, Cardi V et al. (2016) Novel methods to help develop healthier eating habits for eating and weight disorders: a systematic review and meta-analysis. Neurosci Biobehav Rev 61, 132-155.

14. Kakoschke N, Kemps E \& Tiggemann M (2016) Approach bias modification training and consumption: a review of the literature. Addict Behav 64, 21-28.

15. Eberl C, Wiers RW, Pawelczack S et al. (2013) Approach bias modification in alcohol dependence: do clinical effects replicate and for whom does it work best? Dev Cogn Neurosci 4, 38-51.

16. Wiers RW, Eberl C, Rinck M et al. (2011) Retraining automatic action tendencies changes alcoholic patients' approach bias for alcohol and improves treatment outcome. Psychol Sci 22, 490-497.

17. Dickson H, Kavanagh DJ \& MacLeod C (2016) The pulling power of chocolate: effects of approach-avoidance training on approach bias and consumption. Appetite 99, 46-51.

18. Maas J, Keijsers GPJ, Rinck M et al. (2015) Does a dieting goal affect automatic cognitive processes and their trainability? Cogn Ther Res 39, 378-389.

19. Schumacher SE, Kemps E \& Tiggemann M (2016) Bias modification training can alter approach bias and chocolate consumption. Appetite 96, 219-224.

20. Becker D, Jostmann NB, Wiers RW et al. (2015) Approach avoidance training in the eating domain: testing the effectiveness across three single session studies. Appetite 85, 58-65.

21. Brockmeyer T, Hahn C, Reetz C et al. (2015) Approach bias modification in food craving - a proof-of-concept study. Eur Eat Disord Rev 23, 352-360.

22. Wiers RW, Rinck M, Kordts R et al. (2010) Retraining automatic action-tendencies to approach alcohol in hazardous drinkers. Addiction 105, 279-287.

23. Volkow ND, Wang GJ, Tomasi D et al. (2013) Obesity and addiction: neurobiological overlaps. Obes Rev 14, 2-18.

24. Wiers RW, Gladwin TE, Hofmann W et al. (2013) Cognitive bias modification and cognitive control training in addiction and related psychopathology: mechanisms, clinical perspectives, and ways forward. Clin Psychol Sci 1, 192-212.

25. Kromeyer-Hauschild K, Wabitsch M, Kunze K et al. (2001) Perzentile für den Bodymass-Index für das Kindes- und Jugendalter unter Heranziehung verschiedener deutscher Stichproben. Monatsschr Kinderbeilkd 149, 807-818.

26. Borrmann A \& Mensink GBM (2015) Obst- und Gemüsekonsum von Kindern und Jugendlichen in Deutschland:
Ergebnisse der KiGGS-Welle 1. Bundesgesundheitsblatt Gesundheitsforschung Gesundheitsschutz 58, 1005-1014.

27. Baird SO, Rinck M, Rosenfield D et al. (2017) Reducing approach bias to achieve smoking cessation: a pilot randomized placebo-controlled trial. Cogn Ther Res $\mathbf{4 1}$, 662-670.

28. Karpinski A \& Steinman RB (2006) The single category implicit association test as a measure of implicit social cognition. J Pers Soc Psychol 91, 16-32.

29. Blechert J, Meule A, Busch NA et al. (2014) Food-pics: an image database for experimental research on eating and appetite. Front Psychol 5, 617.

30. Cohen J (1988) Statistical Power Analysis for the Behavioral Sciences, 2nd ed. Hillsdale, NJ: Lawrence Erlbaum Associates.

31. Victor A, Elsäßer A, Hommel G et al. (2014) Wie bewertet man die p-Wert-Flut? Dtsch Arztebl 107, 50-56.

32. Huijding J, Field AP, Houwer J de et al. (2009) A behavioral route to dysfunctional representations: the effects of training approach or avoidance tendencies towards novel animals in children. Behav Res Ther 47, 471-477.

33. Kong G, Larsen H, Cavallo DA et al. (2015) Re-training automatic action tendencies to approach cigarettes among adolescent smokers: a pilot study. Am J Drug Alcohol Abuse 41, 425-432.

34. Mensink GB, Kleiser C \& Richter A (2007) Lebensmittelverzehr bei Kindern und Jugendlichen in Deutschland. Ergebnisse des Kinder- und Jugendgesundheitssurveys (KIGGS). Bundesgesundheitsblatt Gesundheitsforschung Gesundheitsschutz 50, 609-623.

35. Lanfer A, Knof K, Barba G et al. (2012) Taste preferences in association with dietary habits and weight status in European children: results from the IDEFICS study. Int $J$ Obes (Lond) 36, 27-34.

36. Craeynest M, Crombez G, Haerens L et al. (2007) Do overweight youngsters like food more than lean peers? Assessing their implicit attitudes with a personalized Implicit association task. Food Qual Prefer 18, 1077-1084.

37. Roefs A \& Jansen A (2002) Implicit and explicit attitudes toward high-fat foods in obesity. J Abnorm Psychol 111, 517-521.

38. Eberl C, Wiers RW, Pawelczack S et al. (2014) Implementation of approach bias retraining in alcoholism - how many sessions are needed? Alcohol Clin Exp Res 38, 587-594.

39. Boendermaker WJ, Prins PJM \& Wiers RW (2015) Cognitive bias modification for adolescents with substance use problems - can serious games help? J Behav Ther Exp Psychiatry 49, 13-20.

40. Connor Gorber S, Tremblay M, Moher D et al. (2007) A comparison of direct vs. self-report measures for assessing height, weight and body mass index: a systematic review. Obes Rev 8, 307-326. 